\title{
Nano spray drying of pharmaceuticals
}

\section{Arpagaus, C.}

NTB University of Applied Sciences of Technology Buchs, Institute for Energy Systems, Werdenbergstrasse 4, 9471 Buchs, Switzerland, +41 817553494

E-mail of the corresponding author: cordin.arpagaus@ntb.ch

\begin{abstract}
Spray drying plays a crucial role in the processing of pharmaceutical products such as pills, capsules, and tablets as it is used to convert drug containing liquids into dried powdered forms. Nano spray drying is in particular used to improve drug formulation by encapsulating active ingredients in polymeric wall materials for protection and delivering the drugs to the right place and time in the body. The nano spray dryer developed in the recent years extends the spectrum of produced powder particles to the submicron- and nanoscale with very narrow size distributions and sample quantities in the milligram scale at high product yields. This enables the economical use of expensive active pharmaceutical ingredients and pure drugs. The present paper explains the concept of nano spray drying and discusses the influence of the main process parameters on the final powder properties like particle size, morphology, encapsulation efficiency, and drug loading. Application results of nano spray drying for the formulation and encapsulation of different drugs are reviewed.
\end{abstract}

Keywords: nano spray drying; pharmaceuticals; drug encapsulation; particle size; powder 


\section{Introduction}

Spray drying is a simple, fast, and scalable drying technology that is well established in the pharmaceutical industry. Many pharmaceutical products such as pills, capsules, and tablets are processed in dried powdered form. In the course of the rapid progress of nanocapsulation techniques, nano spray drying technology has developed to improve the formulation and administration of drugs with solid colloidal particles in the submicron size range. It enables the encapsulation of active ingredients in polymeric wall materials providing enhanced environmental protection (e.g. against oxidation, light, and temperature), stability, handling, storage, and controlled drug release properties. The nanonization and structual change improves the particle solubility and redispersibility of the final drug product in aqueous solutions. This study explains the concept of nano spray drying, the influence of the main process parameters on the powder properties (e.g. particle size, morphology, encapsulation efficiency, drug loading), and discusses different pharmaceutical applications. More detailed information on the formation of nanocapsules by nano spray drying can be found in several review studies [1-12], in particular in a recently published book chapter by Arpagaus et al. [1].

\section{Process Parameters of a Nano Spray Dryer}

Figure 1 shows the functional principle of a nano spray dryer and the adjustable process parameters and formulation variables. The droplet generation is based on vibrating mesh technology ejecting precisely sized droplets into the drying chamber.

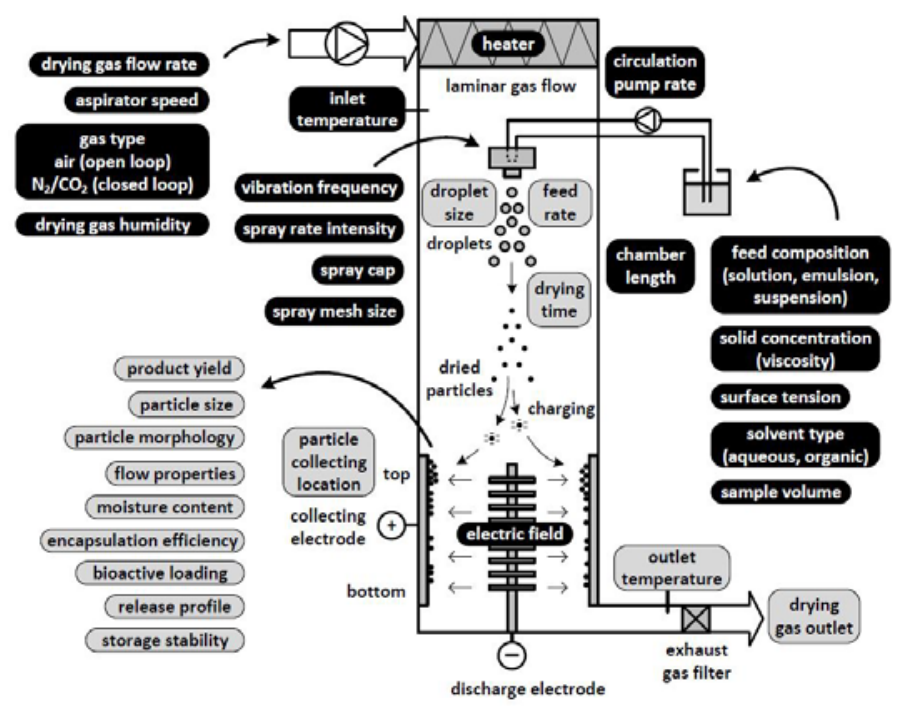

Figure 1 Process parameters and formulation variables for a nano spray dryer [1]. 
With a $4.0 \mu \mathrm{m}$ spray mesh approximately 3 to $8 \mu \mathrm{m}$ water droplets are produced $[1,8]$. The dried particles are captured in an electrostatic particle collector providing high particle collection efficiency. The cooling effect of the evaporating solvent keeps the droplet temperature low, so that heat sensitive products can be dried with negligible degradation. Table 1 povides an overview of the main process parameters and their influence on the final powder product. The thickness of each arrow illustrates the strength of the relationship. The key parameters controlling the final particle size are the spray mesh size and the solid concentration. The submicron size is typically reached when a $4.0 \mu \mathrm{m}$ spray mesh and diluted solutions of 0.1 to $1 \%(\mathrm{w} / \mathrm{v})$ are used.

Table 1 Influence of the main process parameters in nano spray drying ( $\downarrow /$ strong, $t / \downarrow$ weak increasing/decreasing influence, - minimal or no influence) [1].

\begin{tabular}{cccccccc}
\hline $\begin{array}{c}\text { Process } \\
\text { parameter }\end{array}$ & $\begin{array}{c}\text { Outlet } \\
\text { temperature }\end{array}$ & $\begin{array}{c}\text { Droplet } \\
\text { size }\end{array}$ & $\begin{array}{c}\text { Particle } \\
\text { size }\end{array}$ & $\begin{array}{c}\text { Feed } \\
\text { rate }\end{array}$ & $\begin{array}{c}\text { Moisture } \\
\text { content }\end{array}$ & Yield & Stability \\
\hline Drying gas flow rate $\uparrow$ & $\uparrow$ & - & - & - & $\downarrow$ & - & - \\
Drying gas humidity $\uparrow$ & $\uparrow$ & - & - & - & $\uparrow$ & $\downarrow$ & - \\
Inlet temperature $\uparrow$ & $\uparrow$ & - & $\uparrow$ & - & $\downarrow$ & $\uparrow$ & $\downarrow$ \\
Spray mesh size $\uparrow$ & $\downarrow$ & $\uparrow$ & $\uparrow$ & $\uparrow$ & - & - & $\uparrow$ \\
Spray rate intensity $\uparrow$ & $\uparrow$ & $\uparrow$ & $\uparrow$ & $\uparrow$ & $\uparrow$ & - & $\downarrow$ \\
Circulation pump rate $\uparrow$ & - & $\uparrow$ & $\uparrow$ & $\uparrow$ & - & - & $\uparrow$ \\
Solid concentration $\uparrow$ & $\uparrow$ & - & $\uparrow$ & $\downarrow$ & $\downarrow$ & $\uparrow$ & - \\
Surfactant / stabilizer $\uparrow$ & - & $\downarrow$ & $\downarrow$ & $\uparrow$ & - & $\uparrow$ & $\uparrow$ \\
Solvent instead of water & $\uparrow$ & $\downarrow$ & $\downarrow$ & $\uparrow$ & $\downarrow$ & $\uparrow$ & - \\
\hline
\end{tabular}

Depending on the application, an optimized set of process parameters can be found, e.g. by design of experiment studies [1]. Organic solvents like dichloromethane, acetone, ethanol, methanol, acetonitrile, ethylacetate, and mixtures thereof with water are typically used in nano spray drying of pharmaceuticals. The selection is based on the drug solubilization and the encapsulating wall materials, as well as on the required drying temperatures. For acqueous applications, the outlet temperatures range between 28 and $59{ }^{\circ} \mathrm{C}$ [2]. The optimal drying temperatures of for example poly(lactic-co-glycolic acid) (PLGA) dissolved in dichloromethane lies in a range of 29 to $32{ }^{\circ} \mathrm{C}$ [12].

Numerous excipients, dispering agents, binders and stabilizers are applied in drug formulation studies, including water-soluble saccharides (e.g. arabic gum, alginate, chitosan, cyclodextrin, cellulose derivatives, modified starch, maltodextrin, pectin, mannitol, lactose trehalose), proteins (i.e. gelatin, serum albumin, whey protein, sodium caseinate, silk fibroin, leucine), water-soluble synthetic polymers (e.g. poly(vinyl alcohol), poly(ethylene glycol) or poly(acrylic acid) (Carbopol)), hydrophobic synthetic polymers (e.g. PLGA, poly( $\varepsilon-$ caprolactone), poly(vinyl pyrrolidone) (Kollidon), Eudragit), and fats (e.g. stearic acid and glyceryl behenate (Compritol)). 
The morphology of particles prepared by nano spray drying depends on the drying conditions and the feed properties. Dense, hollow, porous, composites, and encapsulated structures (i.e. single-core, multi-core, irregular, or multi-walled) with spherical, wrinkled, shriveled, or even doughnut-like shapes can be obtained [1]. Figure 2 illustrates some examples of nano spray dried particles including salbutamol, albuterol in mannitol, cyclosporine in PLGA, trehalose, $\beta$-galactosidase in trehalose, and bovine serum albumin.
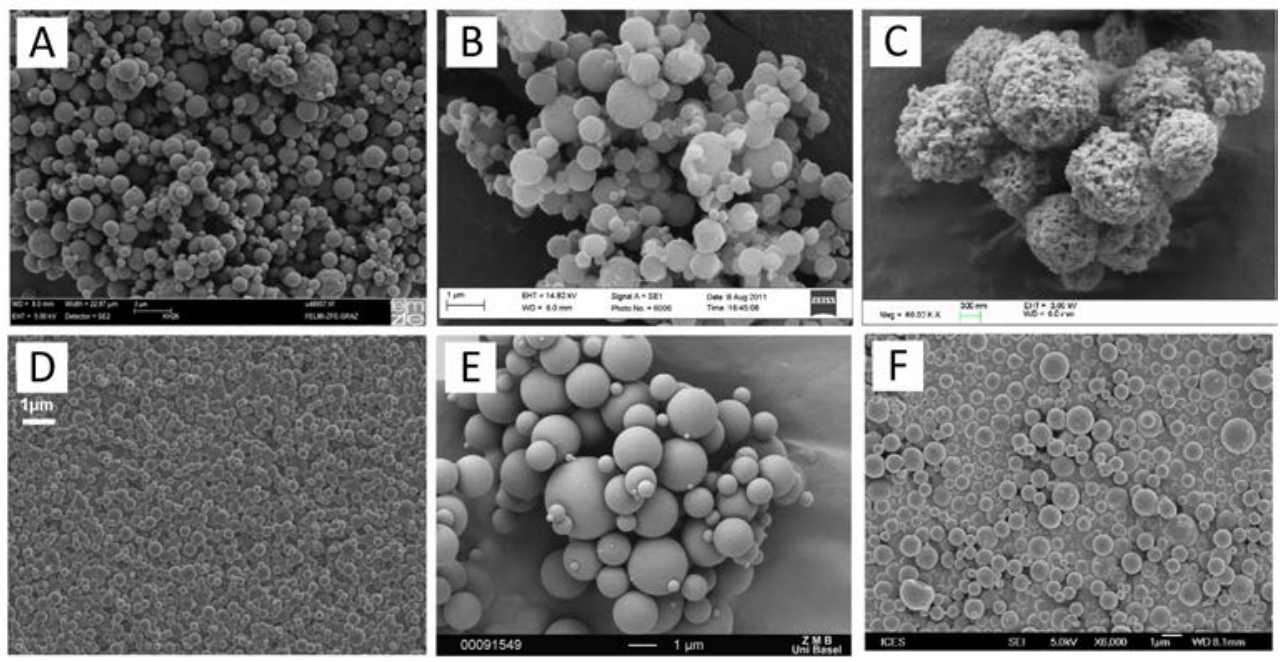

Figure 2 Examples of nano spray dried particles: A: Salbutamol sulfate (1\% solid concentration, nano spray dried at $100{ }^{\circ} \mathrm{C}$ ) [13]; B: Albuterol sulfate in mannitol, L-leucine and poloxamer 188 (30:48:20:2 mixing ratio, 0.5\% in water-ethanol solution (80:20), $70{ }^{\circ} \mathrm{C}$ ) [14]; C: Cyclosporin in PLGA (50:50, $15 \mathrm{kDa}$, dissolved in $\mathrm{DCM}, 29^{\circ} \mathrm{C}$ ) [12]; D: Trehalose with addition of $0.005 \%$ polysorbate $20\left(0.1 \%, 120^{\circ} \mathrm{C}\right)$ [8]; E: $\beta$-galactosidase in trehalose $\left(1: 2\right.$ mixing ratio, $\left.5 \%, 80^{\circ} \mathrm{C}\right)$

[11]; F: Bovine serum albumin with $0.05 \%$ Tween $80\left(0.5 \%, 120^{\circ} \mathrm{C}\right)$ [6].

In general, slow drying leads to more compact particles, while fast and high temperature drying favours the formation of hollow particles with thin shells. Surfactants balance the surface-to-viscous forces inside of the drying droplet and enable the formation of a smooth spherical surface. Composite particles prepared from suspensions and nano spray drying provide a high specific surface area. Most nano spray dried drugs tend to be amorphous due to the too short drying time to form crystalline structures. To prevent recrystallization, the dried powders are stored under dry and controlled conditions. 


\section{Applications of Nano Spray Dried Pharmaceuticals}

The number of publications on nano spray dried pharmaceuticals has risen sharply after the market launch of the Nano Spray Dryer B-90 in 2009 [1]. The formulations contain drugs and excipients for the treatment of various diseases, including

- $\quad$ asthma (e.g. salbutamol, terbutaline, or fluticasone),

- inflammation (e.g. dexamethasone and azithromycin, or pain and fever reducer indomethacin and nimesulide),

- $\quad$ cystic fibrosis (e.g. antibacterial dexketoprofen in Kollidon and Eudragit nanoparticles, or azithromycin in leucine),

- $\quad$ diabetes (e.g. sitagliptin, vildagliptin, and metformin in mucoadhesive Carbopol and gelatin),

- $\quad$ pulmonary arterial hypertension (e.g. resveratrol in poly(caprolactone), or sildenafil in PLGA),

- $\quad$ tuberculosis (e.g. capreomycin or pyrazinamide in L-leucine, or ethambutol mixed with chitosan carrier particles),

- Alzheimer's and Parkinson's diseases (e.g. nanocrystals of calpain inhibitor steroids),

- breast cancer (e.g. simvastatin loaded PLGA particles), or lung cancer (e.g. methotrexate, carboplatin in gelatine, or paclitaxel),

- bacterial infections (e.g. amoxicillin, ciprofloxacin, gatifloxacin, clarithromycin, or levofloxacin),

- fungal infections (e.g. antifungal griseovulfin)

- ophthalmic disorders (e.g. calpain inhibitor nanocrystals or dirithromycin incorporated in Kollidon),

- $\quad$ high blood pressure (e.g. nimodipine in PLGA or pure nicergoline nanoparticles),

- $\quad$ congestive heart failure and edema (e.g. diuretic furosemide).

The drug-loaded nano spray dried particles are administered in various ways underlining the versatility of the applications, such as mainly pulmonary (e.g. optimized respirable particles of 1 to $5 \mu \mathrm{m}$ size) and oral, but also intravenous (e.g. simvastatin in PLGA as cancer chemotherapeutics, antipsychotic clozapine and risperidone in PLGA, or small interfering RNAs loaded in human serum albumin particles to treat genetic disorders), topically as creams to the skin (e.g. anti-inflammatory dexamethasone, antibacterial gentamicin in 
gelatin-pectine, amoxicillin loaded chitosan, antifungal econazole in cyclodextrin, soy isoflavones for skin cancer treatment or as anti-ageing agent), or as nanoparticulate powder (e.g. as a wound dressing during surgery), ophthalmic (e.g. anti-inflammatory steroids in eye drop solutions, or dirithromycin to treat ocular bacterial infections), intraperitoneal (e.g. encapsulated paclitaxel as cytostatic in anticancer therapy), intravesical (e.g. as drug delivery system to treat local bladder diseases), and even cerebral (e.g. with nimodipine in PLGA regulating the dilatation of blood vessels).

Typical experimental process parameters for nano spray drying are listed in Table 2 and can be used as first guess values for applying identical or similar substances. The main organic solvents used to dissolve poorly water soluble drugs are ethanol, acetone, and DCM. With highly diluted solutions containing 0.1 to $1 \%(\mathrm{w} / \mathrm{v})$ solids concentrations, finest solid particles down to $100 \mathrm{~nm}$ can be obtained by nano spray drying.

Table 2 Typical experimental process parameters for nano spray drying of pharmaceuticals.

\begin{tabular}{cccccc|cc}
\hline Solvent & $\begin{array}{c}\text { Inlet drying } \\
\text { temperature } \\
{\left[{ }^{\circ} \mathbf{C}\right]}\end{array}$ & $\begin{array}{c}\text { Outlet drying } \\
\text { temperature } \\
{\left[{ }^{\circ} \mathbf{C}\right]}\end{array}$ & $\begin{array}{c}\text { Drying } \\
\text { gas } \\
\text { type }\end{array}$ & $\begin{array}{c}\text { Drying gas } \\
\text { flow rate } \\
\text { [L/min] }\end{array}$ & $\begin{array}{c}\text { Solid } \\
\text { concentration } \\
\text { [\% } \mathbf{w} / \mathbf{v}]\end{array}$ & $\begin{array}{c}\text { Spray } \\
\text { mesh size } \\
\text { [ } \mathbf{m m}]\end{array}$ & $\begin{array}{c}\text { Feed } \\
\text { Rate } \\
\text { [mL/h] }\end{array}$ \\
\hline Water & 60 to 120 & 30 to 60 & Air & 100 to 140 & & 4.0 & 5 to 25 \\
Ethanol & 50 to 110 & 30 to 50 & Air & 100 to 120 & 0.1 to 10 & 5.5 & 20 to 65 \\
Acetone & 40 to 70 & 25 to 40 & Inert gas & 90 to 120 & & 7.0 & 30 to 160 \\
DCM & 30 to 50 & 20 to 35 & $\left(\mathrm{~N}_{2} / \mathrm{CO}_{2}\right)$ & 80 to 120 & & & \\
\hline
\end{tabular}

Pure drug particles in the nanosize dimensions and the amorphous state offer higher absorption rates and bioavailability, and encourage future developments in this research area. Nanocapsules, with their reduced size and large specific surface area, provide pronounced improvement in controlled drug release and bioavailabilty. This enables the generation of target drug delivery systems. Under optimized conditions uniquely high product yields of about 76 to $96 \%$ can be achieved to process small sample amounts of substances in the range of $10 \mathrm{mg}$ to $2.5 \mathrm{~g}$.

Variations in the yield may occur due to particle depositions around the spray cap and the chamber walls, nozzle blockage, or due to losses during the manual collection of the powder with a rubber spatula.

However, the ability to process small sample amounts makes a nano spray dryer very suitable for testing valuable biological materials such as for example monoclonal antibodies, recombinant proteins, or siRNA-based therapeutics. Moreover, nano spray drying enables the encapsulation of drugs in polymers with high efficiency of over $95 \%$ and adjustable drug loading. 


\section{Conclusions}

Nano spray drying has been successfully applied for a wide range of pharmaceutical applications, such as increasing the bioavailability of poorly soluble drugs by nanoisation and structural modification, as well as the encapsulation of nanoparticles, nanoemulsions and nanosuspensions in biocompatible polymeric wall materials for sustained drug release. Encapsulation efficiencies of over $95 \%$ are achieved by adjustable drug loadings. Smallest sample amounts ranging from $10 \mathrm{mg}$ to $2.5 \mathrm{~g}$ with uniquely high yields of over $95 \%$ can be processed, which enables the economical use of valuable active pharmaceutical ingredients.

Compared to conventional spray drying processes, nano spray drying relies on vibrating mesh technology to produce an ultrafine spray. A highly efficient electrostatic powder collector to extend the size spectrum of separable particles to the nanoscale.

The most important adjustable process parameters are the drying gas temperature, the drying gas flow rate, the spray mesh size, the solvent type, the solids concentration in the feed, and the selection of the corresponding exipients, stabilizers and surfactants. Depending on the application, an optimized set of parameters can be found. Submicron spray dried particles can be formed down to a size of only $100 \mathrm{~nm}$ with diluted solutions of 0.1 to $1 \%(\mathrm{w} / \mathrm{v})$ solids concentration. Different particle morphologies can be created, including dense, hollow and porous particles with spherical, wrinkled, or donut shapes.

The drying process is gentle and contributes to maintaining the stability and activity of heatsensitive materials, such as peptides, proteins, hormones and amino acids. The prepared drug loaded particles are administered in various ways, including pulmonary, oral, intravenous, topically, ophthalmic, intraperitoneal, intravesical, or even cerebral, which underlines the versatility of the nano spray drying technology.

To further explore the potentials of nano spray drying future research should focus on the further commercialization of this technology to an industrial scale. There is an increasing need for scale-up.

The main application trends are in the areas of pulmonary drug delivery, nanotherapeutics, the encapsulation of nanoemulsions with poorly water-soluble active ingredients and the formulation of nanocrystals for a higher bioavailability. 


\section{References}

[1] Arpagaus, C.; John, P.; Collenberg, A. and Rütti, D. Chapter 10: Nanocapsules formation by nano spray drying, in Nanoencapsulation technologies for the food and nutraceutical industries, S.M. Jafari, ed., Elsevier Inc., 2017, 346-401.

[2] Arpagaus, C. A Novel Laboratory-Scale Spray Dryer to Produce Nanoparticles, Drying Technology 2012, 30, 1113-1121.

[3] Arpagaus, C. Nano Spray Dryer B-90: Literature review and applications, best@buchi Information Bulletin, Number 63/2011, 2011, .

[4] Arpagaus, C.; Rütti, D. and Meuri, M. Chapter 18: Enhanced Solubility of Poorly Soluble Drugs Via Spray Drying, in Drug Delivery Strategies for Poorly Water-Soluble Drugs, D. Douroumis and A. Fahr, eds., John Wiley \& Sons, Ltd., 2013, 551-585.

[5] Wong, T.W. and John, P. Advances in Spray Drying Technology for Nanoparticle Formation, in Handbook of Nanoparticles, M. Aliofkhazraei, ed., Springer International Publishing, 2015, $1-16$.

[6] Lee, S.H.; Heng, D.; Ng, W.K.; Chan, H.-K. and Tan, R.B.H. Nano spray drying: A novel method for preparing protein nanoparticles for protein therapy, International Journal of Pharmaceutics 2011, 403, 192-200.

[7] Li, X.; Anton, N.; Arpagaus, C.; Belleteix, F. and Vandamme, T.F. Nanoparticles by spray drying using innovative new technology: The Büchi Nano Spray Dryer B-90, Journal of Controlled Release 2010, 147, 304-310.

[8] Schmid, K.; Arpagaus, C. and Friess, W. Evaluation of the Nano Spray Dryer B-90 for pharmaceutical applications, Pharmaceutical Development and Technology 2011, 16, 287294.

[9] Schmid, K.; Arpagaus, C. and Friess, W. Evaluation of a Vibrating Mesh Spray Dryer for Preparation of Submicron Particles, Respiratory Drug Delivery 2009, 323-326.

[10] Arpagaus, C. Spray Drying R\&D Solutions - BÜCHI’s Nano Spray Dryer: A world novelty in laboratory scale, ONdrugDelivery, March 2010, page 30, available on www.ondrugdelivery.com, 2010, , pp. 40-41.

[11] Bürki, K.; Jeon, I.; Arpagaus, C. and Betz, G. New insights into respirable protein powder preparation using a nano spray dryer, International Journal of Pharmaceutics 2011, 408, 248256.

[12] Schafroth, N.; Arpagaus, C.; Jadhav, U.Y.; Makne, S. and Douroumis, D. Nano and microparticle engineering of water insoluble drugs using a novel spray-drying process, Colloids and Surfaces B: Biointerfaces 2012, 90, 8-15.

[13] Littringer, E.M.; Zellnitz, S.; Hammernik, K.; Adamer, V.; Friedl, H. and Urbanetz, N. a. Spray Drying of Aqueous Salbutamol Sulfate Solutions Using the Nano Spray Dryer B-90 - The Impact of Process Parameters on Particle Size, Drying Technology 2013, 31, 1346-1353.

[14] Son, Y.-J.; Longest, P.W.; Tian, G. and Hindle, M. Evaluation and modification of commercial dry powder inhalers for the aerosolization of a submicrometer excipient enhanced growth (EEG) formulation, European Journal of Pharmaceutical Sciences 2013, 49, 390-399. 